CERN-TH/98-339

IFUM $637 / \mathrm{FT}$

$\mathrm{DTP} / 98 / 76$

hep-ph/9811206

\title{
A Fixed-Order Calculation of THE HEAVY-QUARK FRAgMENTATION FUNCTION IN $e^{+} e^{-}$COLLISIONS
}

\author{
Paolo NASON円, \\ TH Division, CERN, Geneva, Switzerland \\ Carlo OLEARI \\ Department of Physics, University of Durham, Durham, England
}

\begin{abstract}
We use a recently completed $\mathcal{O}\left(\alpha_{s}^{2}\right)$ fixed-order calculation of the heavyflavour production cross section in $e^{+} e^{-}$collisions to compute the heavy-quark fragmentation function. We fit the result of our calculation, convoluted with a Peterson fragmentation function, to available data for charm production, and thus obtain a value for the parameter $\epsilon$ in the Peterson function. We discuss the relevance of mass effects and of subleading terms in our calculation.
\end{abstract}

CERN-TH/98-339

October 1998

\footnotetext{
${ }^{1}$ From November $1^{\text {st }}$ : INFN, Sezione di Milano, Milan, Italy
} 


\section{Introduction}

In this work, we use a recently completed calculation of the $\mathcal{O}\left(\alpha_{s}^{2}\right)$ differential cross section for heavy-quark production in $e^{+} e^{-}$annihilation [1] to compute the heavy-quark fragmentation function at order $\alpha_{s}^{2}$. This calculation should be reliable when the centre-of-mass energy $E$ is not too high. At very high energies, in fact, large logarithms of the ratio $E / m$, where $m$ is the heavy-quark mass, arise at all orders in perturbation theory, and should be resummed. A method for the resummation of the large logarithms at the next-to-leading logarithmic level (NLL) has been developed in Ref. [2]. On the other hand, the fixed-order calculation should be more accurate for moderate values of the energy, since it correctly accounts for mass effects. Furthermore, the NLL calculation correctly accounts for terms proportional to $\alpha_{s}^{2} \log ^{2}(E / m)$ and $\alpha_{s}^{2} \log (E / m)$, but cannot correctly predict the $\alpha_{s}^{2}$ terms that do not carry any logarithmic enhancements, since these terms are of next-to-next-to-leading logarithmic order (NNLL).

Studies of the charm fragmentation function have been performed with relatively recent data in Ref. [3], using a parametrization of the non-perturbative effects based upon the Peterson fragmentation function. From these studies, it was found that the value of the $\epsilon$ parameter is much smaller in NLL fits rather than in leading-log (LL) ones. In this work, we fit the same data sets, using our fixed-order calculation convoluted with a Peterson parametrization of non-perturbative effects, and compare our results with those of Ref. [3]. In order to better understand the differences of the two approaches, we will also consider a fixed-order calculation of the fragmentation function, in which mass-suppressed effects (i.e. effects suppressed by powers of $m / E$ ) and NNLL terms are neglected. This calculation corresponds to a truncation of the NLL formalism at order $\alpha_{s}^{2}$.

\section{Theoretical framework}

We consider the inclusive production of a heavy quark $Q$ of mass $m$

$$
e^{+} e^{-} \rightarrow Z / \gamma(q) \rightarrow Q(p)+X
$$


where $q$ and $p$ are the four-momenta of the intermediate boson and of the final quark. We also introduce the notation

$$
E=\sqrt{q^{2}}, \quad \rho=\frac{4 m^{2}}{q^{2}} .
$$

We will consider two possible definitions of the $x$ variable, one based upon the energy and one based upon the momentum. In the centre-of-mass system, we define

$$
x_{E}=\frac{p^{0}}{p_{\max }^{0}}, \quad x_{p}=\frac{|\vec{p}|}{\left|\vec{p}_{\max }\right|},
$$

with the kinematic ranges

$$
\sqrt{\rho} \leq x_{E} \leq 1, \quad 0 \leq x_{p} \leq 1 .
$$

In terms of invariants, we have

$$
x_{E}=\frac{2 p \cdot q}{q^{2}}, \quad x_{p}=\frac{\sqrt{x_{E}^{2}-\rho}}{\sqrt{1-\rho}} .
$$

Our starting formula will be the fixed-order (FO) cross section for the inclusive production of a heavy-flavoured hadron. It is given by the convolution of the cross section for the inclusive production of a heavy quark, supplemented with a non-perturbative fragmentation function, which describes phenomenologically all the large time phenomena related to the hadronization process

$$
\left.\frac{d \sigma^{H}}{d x_{p}}\left(x_{p}, E, m\right)\right|_{\mathrm{FO}}=\left.\int_{0}^{1} d y d z_{p} \frac{d \sigma}{d z_{p}}\left(z_{p}, E, m\right)\right|_{\mathrm{FO}} P(y, \epsilon) \delta\left(x_{p}-y z_{p}\right),
$$

where $P(y, \epsilon)$ is the Peterson [4] fragmentation function

$$
P(y, \epsilon) \equiv N \frac{y(1-y)^{2}}{\left[(1-y)^{2}+y \epsilon\right]^{2}},
$$

where the normalization factor $N$ is fixed by the condition

$$
\int_{0}^{1} d y P(y, \epsilon)=1
$$

if $P$ refers to the total fragmentation function (i.e., summed over all heavy-flavoured hadron species). In the following, where we will mostly consider $D^{*}$ production, the normalization will be fitted to the data. Notice that we have written the convolution in terms of the momentum fraction, rather than the energy fraction. At large momenta, 
the difference between the two definitions is small. At small momenta, one could choose either approach. Choosing $x_{p}$ seems, however, simpler and more sensible, since it is more conceivable that at small momenta the non-perturbative effects soften the hadron momentum, rather than its mass.

The details of the procedure we followed to perform the calculation will be given in a forthcoming publication. It is, however, quite clear that the heavy-quark inclusive cross section $d \sigma / d z_{p}$ can be computed using the results of Refs. [5, 1]. In order to compute the truncated NLL cross section, we have used the results of Ref. [6], where the NLL evolution equations, with appropriate initial conditions, have been solved exactly up to the second order in the strong coupling constant.

In the present calculation, we have neglected all contributions to the heavy-flavour cross section arising from gluon splitting. These contributions are small at moderate energies, and in general affect the heavy-flavour inclusive cross section at small values of $x$.

\section{Phenomenological results}

Our main results are summarized in Fig. 目, where the fitted, $\mathcal{O}\left(\alpha_{s}^{2}\right)$ fragmentation function is shown together with the ARGUS data for $D^{*+}$ production [7]. The

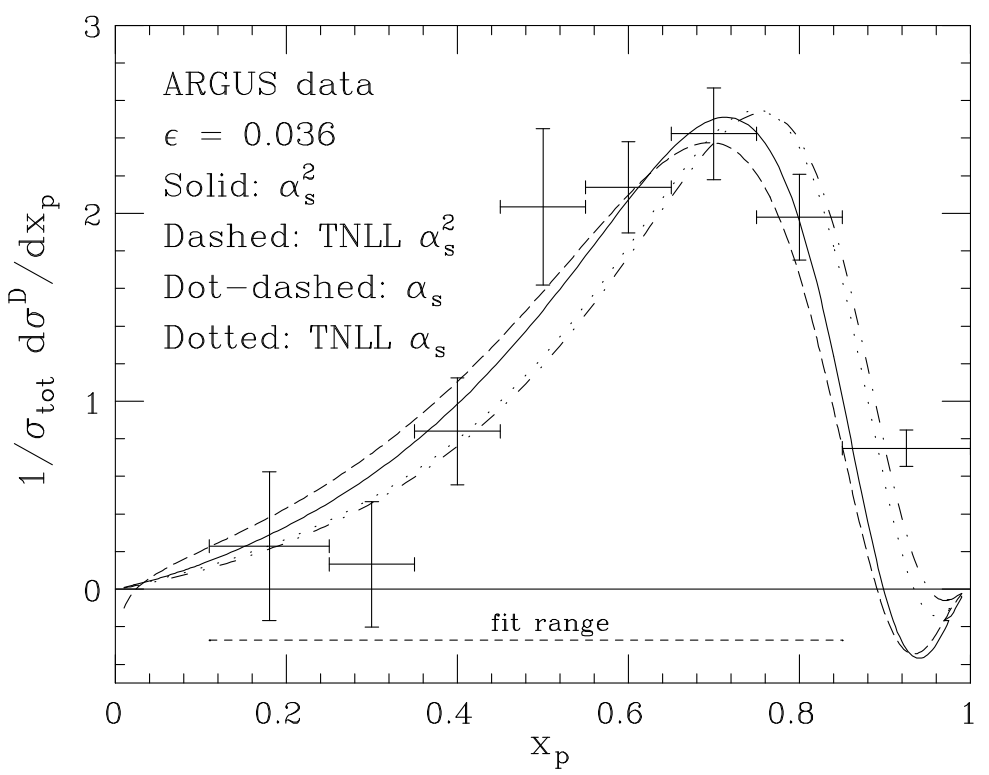

Figure 1: Best fit for the $\mathcal{O}\left(\alpha_{s}^{2}\right)$ fragmentation function at ARGUS. 
free parameters in the fixed-order calculation are the charm-quark mass, which we have fixed at $1.5 \mathrm{GeV}, \Lambda_{\mathrm{QCD}}^{(5)}$, which we have fixed to $200 \mathrm{MeV}$ (corresponding to $\left.\alpha_{s}\left(M_{Z}\right)=0.116\right) \epsilon$, which we have fitted, and the normalization, which we have also fitted. The large- $x$ bin has been excluded from the fit. This is justified, since large logarithms of $(1-x)$ spoil the accuracy of the perturbative expansion in that region. A manifestation of this pathology can be seen in the computed fragmentation function, which becomes negative at large $x$. The result of the fit is $\epsilon=0.036$, with $\chi^{2} /$ dof $=0.853$. In Fig. 11 we also display the $\mathcal{O}\left(\alpha_{s}\right)$ fixed-order result and the truncated expansion of the NLL result (TNLL), both at orders $\alpha_{s}$ and $\alpha_{s}^{2}$. All these curves are obtained with the same value of $\epsilon=0.036$. We see, first of all, that the $\mathcal{O}\left(\alpha_{s}\right)$ fixed-order result is harder than the $\mathcal{O}\left(\alpha_{s}^{2}\right)$ one. In fact, if we attempt to fit the data using the $\mathcal{O}\left(\alpha_{s}\right)$ fixed-order result, we obtain $\epsilon=0.058$, with $\chi^{2} /$ dof $=0.852$. The TNLL, $\mathcal{O}\left(\alpha_{s}\right)$ result differs from the full $\mathcal{O}\left(\alpha_{s}\right)$ one only by terms that are suppressed by powers of the mass over the energy. The curves in the figure seem to indicate that these effects are already quite small for charm at ARGUS energy. The TNLL, $\mathcal{O}\left(\alpha_{s}^{2}\right)$ result differs from the full $\mathcal{O}\left(\alpha_{s}^{2}\right)$ one by terms that are suppressed by powers of the mass over the energy, and by terms of order $\alpha_{s}^{2}$ which are not multiplied by large logarithms of the mass over the energy (NNLL terms). The figure suggests that the presence of these terms makes the fragmentation function harder. Thus, a smaller value of $\epsilon$ would be obtained if we fitted the data using the TNLL $\mathcal{O}\left(\alpha_{s}^{2}\right)$ result.

In Fig. 2 we plot the computed fragmentation function at LEP1 energy, using the same value of $\epsilon=0.036$, together with data from OPAL [ 8 ]. The OPAL data are in terms of $x_{E}$, and we have thus performed the appropriate change of variable in our cross section formulae. The data are arbitrarily normalized. It is apparent from the figure that some evolution effect is present in the fixed-order computation, so that the fragmentation function is softer at higher energy. However, it is not quite as soft as the data would require. If we fit the value of $\epsilon$ to the OPAL data, we get $\epsilon=0.041$, a somewhat larger value than in the ARGUS case. In this fit, besides excluding the large- $x$ region, we should also exclude the small- $x$ bins, since our calculation does not include gluon splitting effects, and these become more significant at high energy. We also plot the TNLL, $\mathcal{O}\left(\alpha_{s}^{2}\right)$ result. We see that at this energy it differs very little from the fixed-order result. It is nevertheless difficult to disentangle mass effects from the NNLL, $\mathcal{O}\left(\alpha_{s}^{2}\right)$ terms. In fact, the former should be reduced by a factor of 10 when going from ARGUS to LEP energies (assuming a linear power law), while the latter should be reduced (roughly) by a factor of 2 , due to the running in $\alpha_{s}^{2}$. The figure 


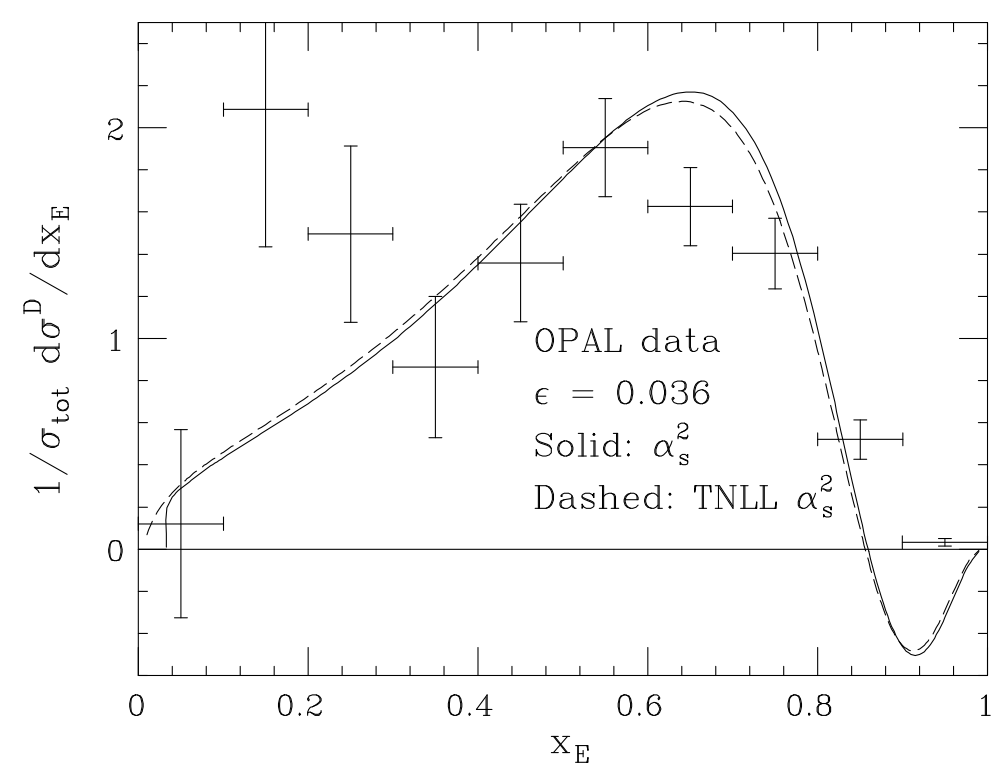

Figure 2: The $\mathcal{O}\left(\alpha_{s}^{2}\right)$ fragmentation function plotted together with OPAL data.

seems to indicate something intermediate between these two values.

We now comment on the differences of our results with those of Cacciari and Greco [3]. These authors have fitted the ARGUS data using a resummed NLL calculation, and found the value $\epsilon=0.02$. This value is considerably smaller than the commonly used value of 0.06 [9], which seems in fact to be appropriate only with leading logarithmic calculations. Our result confirms the fact that, when next-to-leading corrections are introduced, smaller values of $\epsilon$ are needed. On the other hand, our value of $\epsilon$ is larger. This is partly explained by the comparison of our result with OPAL data. We expect that our result will become worse as the energy increases, and conversely, becomes better at lower energies. Since our value of $\epsilon$ increases at higher energies, we expect that it could decrease at lower energies, and thus approach the result of Cacciari and Greco. On the other hand, we have evidence that mass effects do make the fragmentation function harder, and thus require a larger value of $\epsilon$ to fit the data. To state this in a few words, we can say that our result tends to give larger values of $\epsilon$ because it lacks resummation of leading and next-to-leading logarithms beyond the $\mathcal{O}\left(\alpha_{s}^{2}\right)$, while the result of [3] tends to give smaller values of $\epsilon$ because it lacks mass effects. 


\section{Acknowledgements}

We wish to thank M. Cacciari and C. Grab for useful discussions.

\section{References}

[1] P. Nason and C. Oleari, Nucl. Phys. B521 (1998) 237;

C. Oleari, Ph.D. Thesis, hep-ph/9802431.

[2] B. Mele and P. Nason, Nucl. Phys. B361 (1991) 626.

[3] M. Cacciari and M. Greco, Phys. Rev. D55 (1997) 7134.

[4] C. Peterson, D. Schlatter, I. Schmitt and P. M. Zerwas, Phys. Rev. D27 (1983) 105.

[5] P. Nason and B. R. Webber, Nucl. Phys. B421 (1994) 473.

[6] P. Nason and C. Oleari, Phys. Lett. B418 (1998) 199.

[7] H. Albrecht et al. (ARGUS Collaboration), Z. Phys. C52 (1991) 353.

[8] R. Akers et al. (OPAL Collaboration), Z. Phys. C67 (1995) 27.

[9] J. Chrin, Z. Phys. C36 (1987) 163. 